\title{
Chandra and XMM Observations of Galaxy Groups: The Influence of Central AGN at the Low End of the Cluster Mass Scale
}

\author{
J. M. Vrtilek, E. J. O'Sullivan, and L. P. David ${ }^{1}$ \\ ${ }^{1}$ Harvard-Smithsonian Center for Astrophysics, Cambridge, MA 0218, USA
}

Abstract. Chandra and XMM, offering between them high angular resolution, substantial collecting area, and spatially-resolved spectroscopy at good spectral resolution, have given us the means to discover hitherto unanticipated phenomena, in groups as in clusters, and to explore a new set of issues that bring us closer to understanding the formation and evolution of groups and their constituent galaxies: the distribution of heavy elements, the presence of X-ray cavities and their relation to radio observations, the nature of cooling cores, and X-ray signatures of recent galaxy interactions. We here show Chandra and XMM data selected to illustrate recent results regarding some of these themes.

1. NGC 4636: an AGN outburst drives gas mixing?
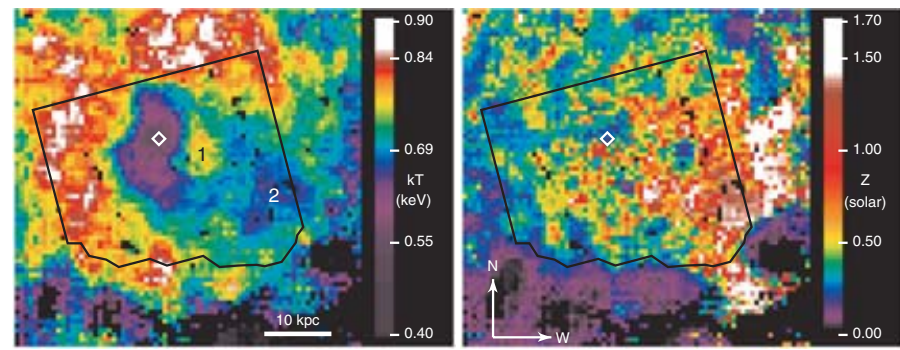

Figure 1. Maps of NGC 4636 from XMM data showing (left) temperature (in keV) and (right) abundance (in solar units). The images suggest that cool, high-abundance gas has been drawn from the core in earlier AGN outbursts.

2. NGC 3411: temperature anomalies from recent AGN reheating?

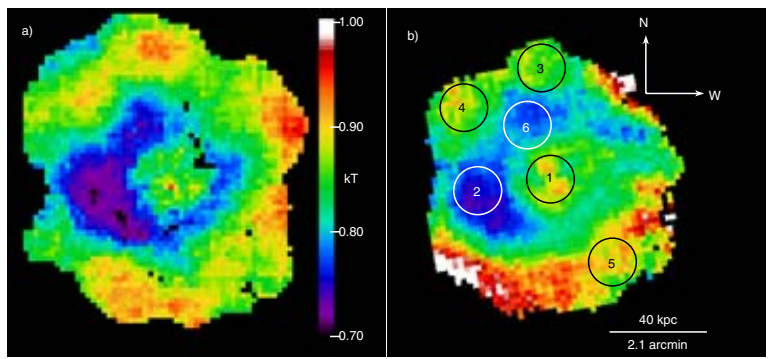

Figure 2. Temperature maps of NGC 3411 from (left) XMM and (right) Chandra data showing an unusual profile: a hot inner core surrounded by a cool shell of gas within the larger group halo. Previous AGN activity has left both heating and cooling effects visible.

\section{References}

Jones, C., et al. 2002, ApJ, 567, L115.

Ohishi, A., Kawano, M., \& Fukazawa, Y. 2003, PASJ, 55, 819.

Sullivan, E. O., Vrtilek, J. M., Harris, D. E., \& Ponman, T. J. 2006, Ap.J., submitted.

Sullivan, E. O., Vrtilek, J. M., \& Kempner, J. C. 2005, ApJ, 624, L77. 